University of Nebraska - Lincoln

DigitalCommons@University of Nebraska - Lincoln

3-1-1998

\title{
Temporal Contrast in Ti:Sapphire Lasers: Characterization and Control
}

\author{
Marc Nantel \\ University of Michigan - Ann Arbor \\ Jiro Itatani \\ University of Tokyo, Minato-ku, Tokyo \\ An-Chun Tien \\ University of Michigan - Ann Arbor \\ Jerome Faure \\ University of Michigan - Ann Arbor \\ Daniel Kaplan \\ Alliage Inc., 75005 Paris, France \\ See next page for additional authors
}

Follow this and additional works at: https://digitalcommons.unl.edu/physicsumstadter

Part of the Physics Commons

Nantel, Marc; Itatani, Jiro; Tien, An-Chun; Faure, Jerome; Kaplan, Daniel; Bouvier, Marcel; Buma, Takashi; Rompay, Paul Van; Nees, John; Pronko, Peter P.; Umstadter, Donald P.; and Mourou, Gerald A., "Temporal Contrast in Ti:Sapphire Lasers: Characterization and Control" (1998). Donald Umstadter Publications. 14. https://digitalcommons.unl.edu/physicsumstadter/14

This Article is brought to you for free and open access by the Research Papers in Physics and Astronomy at DigitalCommons@University of Nebraska - Lincoln. It has been accepted for inclusion in Donald Umstadter Publications by an authorized administrator of DigitalCommons@University of Nebraska - Lincoln. 


\section{Authors}

Marc Nantel, Jiro Itatani, An-Chun Tien, Jerome Faure, Daniel Kaplan, Marcel Bouvier, Takashi Buma, Paul Van Rompay, John Nees, Peter P. Pronko, Donald P. Umstadter, and Gerald A. Mourou 


\title{
Temporal Contrast in Ti:Sapphire Lasers: Characterization and Control
}

\author{
Marc Nantel, Jiro Itatani, An-Chun Tien, Jérôme Faure, Daniel Kaplan, Marcel Bouvier, \\ Takashi Buma, Paul Van Rompay, John Nees, Peter P. Pronko, \\ Donald Umstadter, and Gérard A. Mourou, Fellow, IEEE
}

\begin{abstract}
As ultrafast lasers achieve ever higher focused intensities on target, the problem of ensuring a clean laser-solid interaction becomes more pressing. In this paper, we give concrete examples of the deleterious effects of low-contrast interactions, and address the problem of subpicosecond laser intensity contrast ratio on both characterization and control fronts. We present the new technique of high-dynamic-range plasma-shuttered streak camera contrast measurement, as well as two efficient and relatively inexpensive ways of improving the contrast of short pulse lasers without sacrificing on the output energy: a double-pass Pockels cell (PC), and clean high-energy-pulse seeding of the regenerative amplifier.
\end{abstract}

Index Terms-Amplified spontaneous emission, chirped-pulse amplification, intensity contrast ratio, pulse cleaning, terawatt lasers.

\section{INTRODUCTION}

$\mathbf{T}$ HE ADVENT of chirped-pulse amplification (CPA) [1] and subsequent amplification of subpicosecond pulses to higher energies [2]-[5] has brought high-field-science back to university laboratories. Focusing such lasers in gas targets brings high-harmonics generation [6]-[8], electron acceleration [9]-[12], and other interesting physical phenomena such as relativistic self-focusing [13] and high-energy ions from exploding clusters [14]. In solid-target interactions, table-top subpicosecond laser systems have reached focused intensities of more than $10^{19} \mathrm{~W} / \mathrm{cm}^{2}$ on target, delivered on a timescale much shorter than the hydrodynamical motion of the expanding plasmas. This impulsive energy deposition has made possible ultrafast X-ray sources [15]-[18], high-harmonics generation from solid targets [19] and ion acceleration from plasma bilayers [20].

Manuscript received October 20, 1997; revised March 2, 1998. This work was supported by the National Science Foundation through the Center for Ultrafast Optical Science under Grant STC PHY 8920 108, by the Department of Energy under Contract DE-FG02-96ER14685, and by the U.S. Air Force under Contract F49620-95-1-0474. The work of J. Itatani was supported by IMRA, America Inc. The work of M. Nantel was supported in part by the FCAR fund.

M. Nantel was with the Center for Ultrafast Optical Science (CUOS), University of Michigan, Ann Arbor, MI 48109-2099 USA. He is now with Photonics Research Ontario, ON, M5S 1A7 Canada.

A.-C. Tien, J. Faure, T. Buma, P. Van Rompay, J. Nees, P. P. Pronko, D. Umstadter, and G. A. Mourou are with the Center for Ultrafast Optical Science (CUOS), University of Michigan, Ann Arbor, MI 48109-2099 USA

J. Itatani was with the Institute for Solid State Physics of the University of Tokyo, Minato-ku, Tokyo 106, Japan. He is now with the Laser Technology Laboratory, Institute for Physical and Chemical Research (RIKEN), Wako, Saitama 351-0198, Japan.

D. Kaplan is with Alliage Inc., 75005 Paris, France.

M. Bouvier is with Medox Electro-Optics Inc., Ann Arbor, MI 48108 USA. Publisher Item Identifier S 1077-260X(98)03842-8.
In the case of solid-target interactions, a major concern is that of unwanted energy preceding the pulse, either in the form of incorrect recompression of the stretched pulses, of prepulses from the regenerative amplifier or of an amplified spontaneous emission (ASE) background. A common figure to quantify this unwanted energy is the intensity contrast ratio (ICR), which is the ratio of the peak pulse intensity to that of the prepulses or background. The typical ICR for subpicosecond lasers at $1.06 \mu \mathrm{m}$ (glass laser) or 780 $\mathrm{nm}$ (Ti:sapphire laser) is on the order of $10^{4}-10^{6}$, and the background can therefore contain a significant portion of the pulse energy. In the case of a 100-fs pulse for example, an ASE background of contrast $10^{5}$ and 10-ns duration (typical roundtrip time of a regenerative amplifier) will contain as much energy as the main pulse itself. When that pulse is focused to $10^{18} \mathrm{~W} / \mathrm{cm}^{2}$, the target will see an intensity of $10^{13} \mathrm{~W} / \mathrm{cm}^{2}$ from the ASE alone. Therefore, there will be a formation of an expanding preplasma before the arrival of the main pulse, which will no longer interact with a solid target. This can have damaging effects on the quality of the interaction and on the physical phenomenon being studied. It is, therefore, crucial to both characterize and control the prepulses and background accompanying the main laser pulse. In the next section, we give three examples of ultrashort laser applications where the laser contrast plays a significant role: in the generation and probing of highly compressed materials, in the production of energetic ions from solid targets, and in the development of laser-triggered jitter-free detectors. In Section III, we present contrast measurements of the CUOS $10-\mathrm{Hz} 100-\mathrm{fs}$ terawatt laser obtained with a new method using a high-dynamic-range plasma-shuttered visible streak camera [21]. This technique is particularly well-adapted to measurements over nanosecondscale durations. We then present in Section IV two new techniques to reduce the background of subpicosecond lasers using: 1) a double-pass, high-contrast Pockels cell (PC) and 2) high-energy clean-seed-pulse injection into a regenerative amplifier [22]. We summarize in the conclusion at Section V.

\section{EFFECT OF PUlSE CONTRAST ON SOLID-TARGET INTERACTIONS}

For solid-target interactions, the effective laser pulse duration is not the usual full-width at half-maximum (FWHM) of the pulse intensity. Indeed, the only part of the laser pulse that will interact with a solid target is that which precedes and includes the damage threshold fluence. However short the pulse FWHM, if it sits atop a 10-ns pedestal that can create 
a preplasma, its duration might as well be $10 \mathrm{~ns}$. By the time the main pulse hits the target, it will interact with a long scalelength plasma and deposit its energy at relatively low densities, no longer making this a solid-target/subpicosecond-laser experiment. Preplasma formation from the background can occur at intensities ranging from $10^{8}-10^{13}-\mathrm{W} / \mathrm{cm}^{2}$, depending on the pulse duration (from nanosecond to subpicosecond) [23], [24]. With focused main pulse intensities now routinely in the $10^{15}-10^{19} \mathrm{~W} / \mathrm{cm}^{2}$ range, an ICR of $10^{4}-10^{6}$ is no longer sufficient, as is illustrated by the three following examples.

\section{A. The Generation and Probing of Highly Compressed Solids}

Studies of highly compressed solids are particularly relevant to the understanding of dense stars [26], inertial confinement fusion pellet compression [26], [27], and the equation-of-state of high-density materials [28], [29]. In the laboratory, one can use a laser to create a shock wave and compress a target with tens-of-megabars pressures while probing its thermodynamical properties under these extreme conditions with laser-produced X-ray backlighter sources [28]-[30] or optical interferometric techniques [31]. When compressing the material with nanosecond pulses, one has to deal with the extra complication of there being significant hydrodynamic motion of the target during the laser pulse [28] unless particular care is taken to properly tamp the shocked material [29]. Using a subpicosecond laser removes this concern by depositing the energy impulsively, in a delta-function-like fashion. One is, therefore, left with the much simpler problem of a plasma expansion in the vacuum forming a shock wave in the solid, without further energy input during the measurement [30], [31]. Furthermore, for shortpulse conditions, calculations predict up to gigabar-pressure shock waves lasting picoseconds in duration [32], [33] for high-intensity interactions.

The contrast problem in short-pulse Ti:sapphire lasers restricts them to meaningful solid-target interactions for intensities of $10^{14} \mathrm{~W} / \mathrm{cm}^{2}$ or less [30], [31], making it impossible so far to reach these promised gigabar pressures. For the case of X-ray backlighting shock probing, low contrast impedes two aspects of the experiment: first, the launch of the shock wave and secondly, the generation of the picosecond X-ray source whose duration sets the temporal resolution of the measurement. We observed this in an experiment performed at CUOS which measured the X-ray absorption spectrum of compressed aluminum around the $L$ photoabsorption edge [30]. The $50-\mathrm{mJ}$ pulses at $780 \mathrm{~nm}$ of the CUOS $10-\mathrm{Hz}$ 100 -fs laser were split such that $90 \%$ of the energy was used to generate a bright broad-band short X-ray source in the $100-200-\AA$ range from a gold target. The other $10 \%$ was focused on a $1000-\AA$ film of aluminum. The measurement is a pump-probe acquisition, where the pump is the shocklaunching laser pulse and the probe is the X-ray backlighter. The ICR for this experiment was measured to be $10^{5}$ in the nanosecond timescale with a third-order autocorrelator. The solid-target laser-produced X-ray source duration depends critically on the density at which the laser light is absorbed by the gold target, as it is governed by atomic rates proportional to the electron density in the plasma [18]. The presence

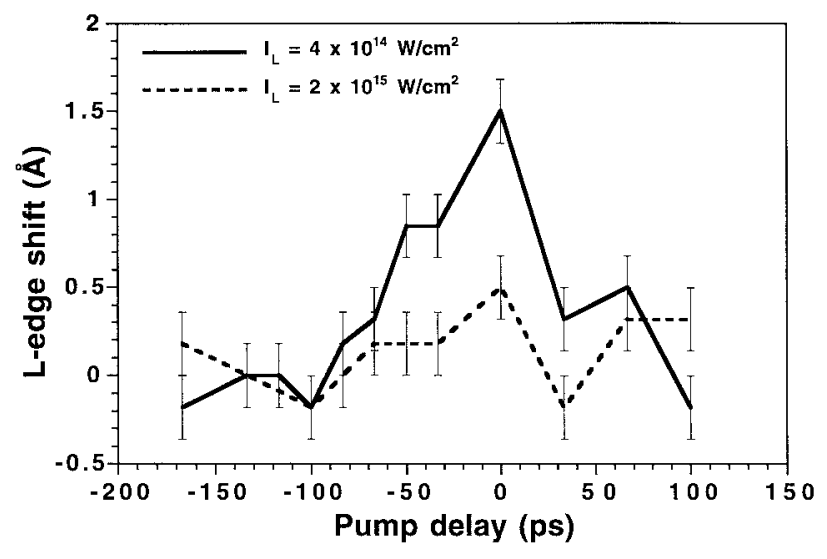

Fig. 1. Shift of the $L$ photoabsorption edge of a $1000-\AA$ aluminum film as a function of the pump-probe delay. Negative values on the horizontal axis refer to the X-ray backlighter arriving before the laser pump. A maximum shift of $1.5 \AA$ is observed with pump intensity of $4 \times 10^{14} \mathrm{~W} / \mathrm{cm}^{2}$ (solid line) from a perturbation of the electronic structure in the aluminum as it is compressed by the shock wave launched by the pump laser. A much reduced shift is observed at higher pump intensity $\left(2 \times 10^{15} \mathrm{~W} / \mathrm{cm}^{2}\right.$, dashed line)

of a preplasma "slows down" the atomic rates relevant to the emission of the X-rays, making the source longer [18], [34]. In a pump-probe measurement, this will degrade the temporal resolution. In the example shown here, the X-ray source duration was limited to $50 \mathrm{ps}$ for that very reason. As the aluminum target compresses under the shock wave generated by the pump laser pulse, the $L$-edge absorption feature at $171 \AA$ shifts to higher wavelength (redshift) due to the perturbations in the atomic structure of the atoms under compression. Fig. 1 shows the amount of shift as a function of the time delay between the laser pump and the $\mathrm{X}$-ray probe, with negative delays corresponding to the X-ray probe arriving before the pump, through an unperturbed target; at time $0 \mathrm{ps}$, the pump and the probe arrived at the target simultaneously. The pump laser intensity was varied from $4 \times 10^{14} \mathrm{~W} / \mathrm{cm}^{2}$ (solid line) to $2 \times 10^{15} \mathrm{~W} / \mathrm{cm}^{2}$ (dashed line) . From the solid line, one can readily see the redshift of the edge during the compression and the return to its original position as the material relaxes. The maximum shift of about $1.5 \AA$ corresponds to a compression of aluminum by a factor 1.4 [30]. Theory predicts that higher laser intensities on target should give a stronger shock wave and, hence, a larger shift of the edge. The experimental edge shift in Fig. 1 shows a different trend, with the larger shift present in the lower intensity case. This surprising result at higher intensity is attributed to the creation of a preplasma by the ASE in the laser pulse, which prevented the proper coupling of the main pulse energy into a shock wave in the target. Hence, to generate high-pressure shock waves and diagnose them with picosecond temporal resolution requires a much better laser contrast.

\section{B. The Production of Energetic Ions}

Another field where solid-target interaction is emerging is in the production of fast ions from laser-ablation plumes. Much theoretical work [35]-[38] and relatively less experimental work [39]-[41], [20] has been done on the subject. The theory states that fast electrons accelerated out of the solid target 


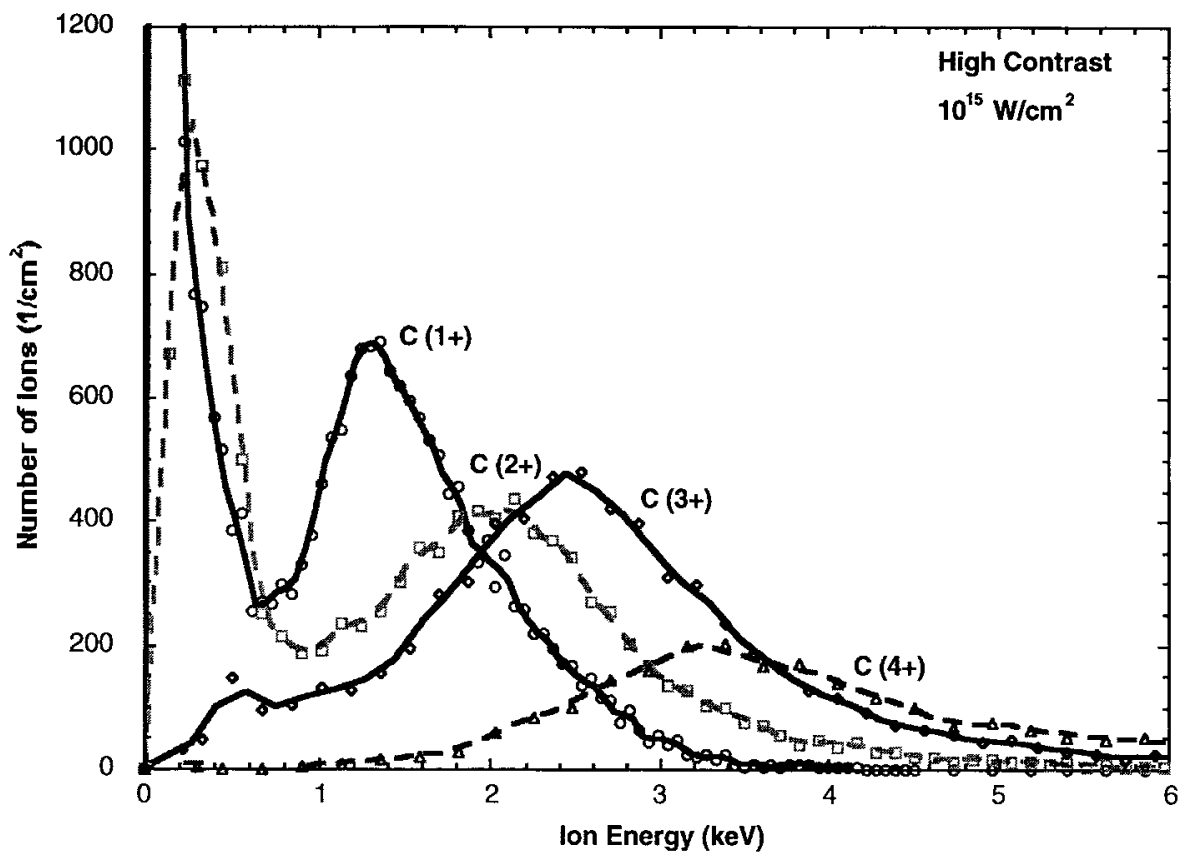

Fig. 2. Ion-energy distributions separated by charge state for carbon ablation with $100-\mathrm{fs} 10^{15} \mathrm{~W} / \mathrm{cm}^{2}$ laser pulses $\left(\mathrm{C}^{+1}\right.$ peaks at 4211 ions/cm $)$. The low-energy hump in the distribution can be explained by a hydrodynamic expansion process, and the high-energy part of the distribution by a bilayer ion-accelerated process.

by the ultrashort laser pulse will form a charge separation between the then positively charged target surface and the negatively charged electron cloud in the vacuum. This bilayer will act like a capacitor and set up an electric field that can accelerate the ions from the target to multikilo-electron volt or even mega-electron volt energies. This effect only works if both "capacitor plates" are well defined, and thus necessitate a clean laser-solid interaction. Any long-scale-length preplasma would destroy the charge separation before it even begins.

In an experiment performed at CUOS with the $10-\mathrm{Hz} 100-$ fs laser, we detected and analyzed the energy distributions of such energetic ions accelerated from carbon plasmas. The laser pulses at $780 \mathrm{~nm}$ were focused to an intensity of $10^{15} \mathrm{~W} / \mathrm{cm}^{2}$ on a solid carbon target with an off-axis parabola, and the ion content of the ablation plume was analyzed with a timeresolved spherical-sector electrostatic ion spectrometer [20]. Ion spectra were collected at the end of the spectrometer on a multichannel plate for a given energy-to-charge $(E / q)$ ratio setting of the spectrometer, with the different charge states previously separated in a time-of-flight drift tube. From a series of spectra taken at different $E / q$ settings, one can reconstruct the ion energy distributions, as shown in Fig. 2. The distributions for each charge state show a bimodal shape. The low-energy hump $(E<1 \mathrm{keV})$ has been identified as the thermal part due to hydrodynamic expansion of the plasma, which cannot account for the charge-dependent high-energy hump. The latter is attributed to the plasma bilayer acceleration mechanism, and good agreement for the high-energy peak positions was obtained with a simple capacitor-acceleration model [20].

The effect of varying the contrast of the laser pulse on the production of these energetic ions is shown in Fig. 3. The energy distributions of $\mathrm{C}^{+2}$ ions are plotted for a fixed

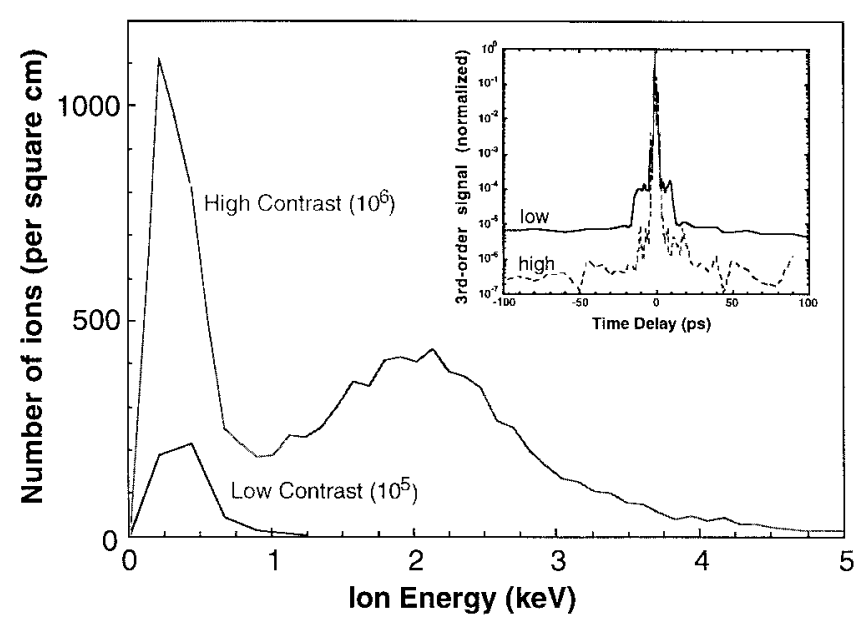

Fig. 3. Ion distribution of $\mathrm{C}^{+2}$ for laser intensity contrast ratios of $10^{5}$ ("low contrast") and $10^{6}$ ("high contrast") at a fixed laser intensity of $10^{15} \mathrm{~W} / \mathrm{cm}^{2}$. The inset shows the measured contrast for the corresponding distributions. The strong dependence of the ion energy distribution on laser contrast is clearly visible, particularly in the absence of the high-energy distribution in the low-contrast case.

laser intensity of $10^{15} \mathrm{~W} / \mathrm{cm}^{2}$, but for different ICR of the laser; the inset shows the third-order autocorrelation of the two different pulses. In the "high-contrast" case (ICR $\approx 5 \times 10^{6}$ ), the energy distribution displays both thermal and accelerated humps, while in the "low-contrast" case (ICR $\left.\approx 2 \times 10^{5}\right)$ only the thermal part of the distribution remains: the creation of a preplasma by the low-contrast laser pulse has prohibited the formation of the accelerating bilayer. The energetic ions obtained in the high-contrast case are interesting for their relevance to the understanding of various laser absorption mechanisms, fast electron generation and thin-film deposition [42], but also as a potential fast-ion source if scalable to 
higher energies. These higher ion energies will come from higher laser intensities on target, which will only be possible with a very good ICR, as the data shown above indicates that a background intensity of $5 \times 10^{9} \mathrm{~W} / \mathrm{cm}^{2}$ is enough to dramatically affect fast-ion production.

\section{The Development of Laser-Triggered Jitter-Free Detectors}

Finally, with the availability of high-repetition-rate shortpulse lasers comes the possibility of averaging multiple laser shots for time-resolved measurements with a high signal-tonoise ratio (SNR). Indeed, recent progress in fast photoconductive switching has made it possible to optically trigger streak cameras with the same laser that is used for the interaction experiment, thereby reducing the shot-to-shot jitter of the camera from tens of picoseconds to subpicosecond values [43]-[47]. Typically, the photoconductive switch is inserted between a power supply and the sweep plates of the camera. The switch has a very high resistivity when not illuminated and it can hold the bias applied by the power supply. When a laser pulse hits the switch (in synchronization with the experimental light hitting the camera's photocathode), its resistivity goes down and a voltage ramp is seen by the plates of the streak camera, which then sweep the electron beam created by the camera's photocathode. If the voltage ramp is kept stable on every shot, the jitter on the measurement is reduced appreciably compared to the more classical case where avalanche transistors are used.

The switch system response to laser light is linear at low intensity and saturates at high intensity when the switch resistance becomes comparable to the circuit impedance. The switch is usually operated in the saturation regime to minimize the jitter produced by shot-to-shot variations in laser intensity. However, under these conditions the switch response to the ASE has a contrast ratio to the main pulse response that is lower than the laser intensity contrast ratio. This degradation induces ASE-related jitter in the measurement. Saturable absorbers can be used in front of the switch to reduce this problem [44], but the optimal solution is still to operate with a better laser contrast ratio.

\section{Measuring the Contrast on the NANOSECOND Scale}

The four main causes of contrast problems in CPA lasers are: 1) prepulses due to the finite extinction ratio of the polarizing optics and the misalignment of the birefringent materials (nanosecond timescale); 2) shoulders and pedestals arising from spectral clipping and phase distortion in the system (tens of picosecond timescale); 3) a typical ICR of $10^{6}$ from Kerr-lens mode-locked Ti:sapphire oscillators (for at least several picoseconds) [48], [49]; and 4) considerable ASE from the large amplification ratio in the whole laser (nanosecond timescale). It is, therefore, important to measure the ICR of a laser not only in the vicinity of the subpicosecond pulse, but in the multinanosecond range to have a complete history of the laser pulse. Third-order autocorrelations are well-adapted for high-dynamic-range measurements near the main pulse, but extending them to longer timescales can prohibitively lenghten the procedure time. Streak cameras allow to rapidly measure a

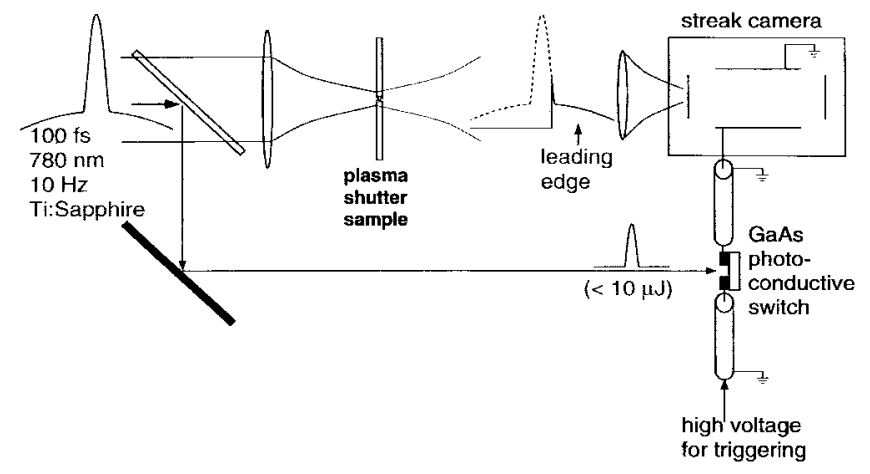

Fig. 4. Experimental setup for plasma-shuttered signal-averaged streak camera.

signal over a long time duration, but have a dynamic range of about a hundred for a given gain setting, which is far less than the required range of $10^{6}-10^{8}$. It would therefore be advantageous to find a way to extend the dynamic range of the streak camera such that it can simultaneously record the background and the main pulse. We accomplished this by using two tricks: plasma shuttering to reduce the main pulse intensity, and multishot accumulation to increase the signal of the background and the overall SNR.

\section{A. High-Dynamic-Range Plasma-Shuttered Streak Camera}

As mentioned earlier in Section II-C, recent advances in photoconductive switch technology makes possible the use of visible and $\mathrm{X}$-ray streak cameras in near-jitter-free mode. In addition to permit the detection of weak signals through shot accumulation, this technique increases the signal-to-noise ratio by a factor $\sqrt{N}$, where $N$ is the number of shots accumulated, while it conserves the time resolution of the camera. This can de facto increase the dynamic range of the streak camera arbitrarily, provided, as in the case of an ICR measurement for example, that the detector remains unsaturated by the main pulse when the background is detectable. To ensure this, one effectively needs an inverse saturable absorber: a filter that would let through the low-intensity background in front of the pulse but attenuate the main pulse itself.

Laser-induced breakdown in transparent media can provide the necessary shuttering effect [24], [50]-[54]. The lowintensity background in front of the pulse goes through the transparent medium unaffected, while the intense ultrashort laser pulse converts the material into a dense plasma. A high enough laser pulse fluence can lead to the formation of a critical surface in the plasma-where the plasma wave frequency exceeds the laser frequency-making the plasma highly reflective. The transmitted laser energy is then greatly reduced and the plasma shutter can serve as a peak-reduction device to prevent the camera from being saturated or damaged. We demonstrate below that the combination of both photoconductive switching and plasma-shuttering can increase the dynamic range of a visible streak camera to up to $10^{9}$, and we use this technique to measure the ICR of the $10-\mathrm{Hz} 100-\mathrm{fs}$ laser [21].

Fig. 4 shows the experimental setup. We used a picosecond Thomson-CSF TSN-906 visible streak camera modified by 


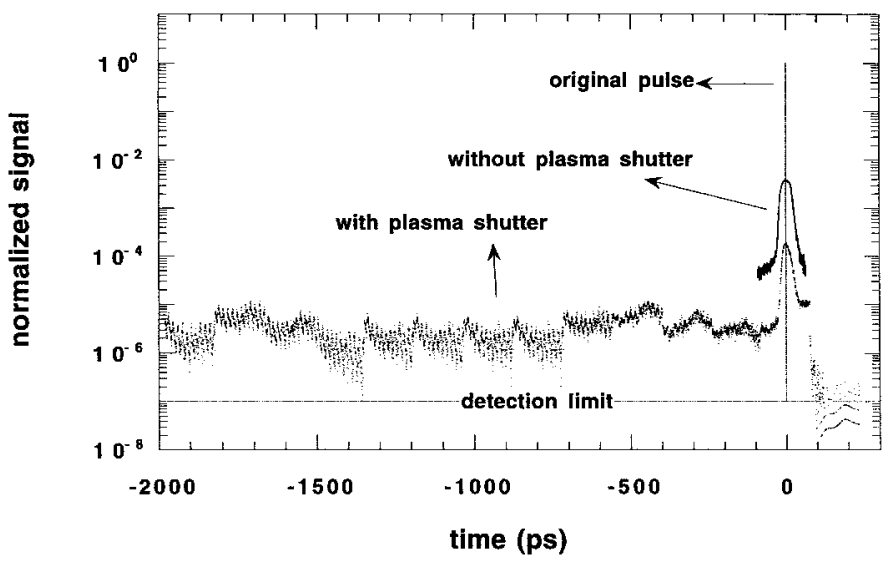

Fig. 5. Recorded signals for the plasma-shuttered streak camera contrast measurement. The curves are normalized to the peak intensity of the original 100 -fs pulse. Measurements were accumulated of 300 laser shots with the signal-averaged sweeping mode of the streak camera. The thin vertical line labeled "original pulse" at time zero represents the 100-fs laser pulse used in the experiment. The curves labeled "without plasma shutter" and "with plasma shutter" are the measurements with low input energy (i.e., no breakdown on the sample) and with full input energy (plasma shutter breakdown on the sample), respectively.

Medox Electro-Optics, Inc. A small fraction of the 780$\mathrm{nm}$ laser pulse illuminated the photoconductive switch and triggered the sweep of the camera, while $300 \mu \mathrm{J}$ was focused in a $150-\mu \mathrm{m}$-thick fused silica plate to generate the plasma shutter. The plate was rastered during the experiment to ensure a fresh spot for each shot. The focal spot of the laser on the plate was magnified and imaged onto a $100-\mu \mathrm{m}$ pinhole in front of the streak camera slit to ensure that the entire apertured area comes from the plasma-shuttered region of the laser-solid interaction.

In Fig. 5, we plot the measured signal normalized to the peak of the laser pulse. The thin vertical line labeled "original pulse" at time zero represents the 100-fs laser pulse. The curves labeled "with plasma shutter" ("without plasma shutter") are the accumulation of 300 laser shots with input energy attenuated (not attenuated) by the plasma shutter. In the case where the plasma shutter was not used, the intensity of the laser pulse was attenuated by neutral density filters to prohibit laser breakdown on the sample, and the ASE background level was undetectable with the streak camera; the detected signal is corrected for these filters and is directly comparable to the other curves in Fig. 5. The attenuation factor obtained from the plasma shutter was 23 , which compares well with the theoretical calculation of 19 based on the damage threshold fluence and the geometry of the imaging [21]. The measured temporal width of the main pulse was 25-ps FWHM, due to a combination of space-charge broadening in the streak camera and residual jitter due to the shotto-shot laser energy fluctuations $( \pm 5 \%)$. The space-charge broadening was a consequence of operating the camera at a high signal level to enhance its dynamic range, and for the sake of simplifying the experiment we tolerated a similar level of jitter-related broadening which could have been reduced if needed to less than a picosecond with proper use of a saturable absorber in front of the switch. Since the goal of this experiment was to characterize the ASE background of the pulse in the nanosecond timescale, the 25-ps temporal resolution was considered adequate. The measured ICR is about $10^{5}$, in agreement with a third-order autocorrelation of the laser system in the same configuration (see Section IVB). The dynamic range of the measurement is given by the lowest signal level measured at $10^{-7}$ after the main pulse. The abrupt drop of signal after the main pulse is evidence that the sample used in the experiment becomes opaque after the plasma density reached the breakdown level during the main pulse. The dynamic range of the technique can be pushed further by optimizing the plasma imaging geometry (using a smaller pinhole) and by replacing the fused silica plate by a material with a lower damage threshold (so the reflective critical plasma density is achieve earlier into the main pulse for less transmitted light). Preliminary experiments with better imaging and a low-damage-threshold photopolymer confirm that the plasma-shuttered streak camera system can reach a dynamic range of $10^{9}$.

\section{CONTRASt ImPRovement Methods}

Characterizing the ICR is only half the battle, one must also improve it. The figure of $10^{4}-10^{6}$ given earlier as a typical ICR for glass and Ti:sapphire lasers is representative of most, but a few groups have focused particular attention on improving the ICR of their lasers [55], [56].

Incomplete recompression can always be corrected with proper alignement of the system and various third- and fourthorder compensation tricks [57]-[60], but prepulses and ASE are sometimes more problematic. Frequency doubling takes advantage of the nonlinearity of the doubling process to suppress the low-intensity background, and more-or-less squares the ICR to values better than $10^{10}$. For glass lasers, this option is a viable one because of the relatively long pulse durations $(\approx 450 \mathrm{fs})$ : one can use a thick, efficient doubling crystal without significantly affecting the pulse duration. Conversion efficiencies of up to $80 \%$ have been reported in this case [61]. For shorter Ti:sapphire pulses ( $<100 \mathrm{fs}$ ), thick crystals become problematic and doubling efficiencies are usually on the order of $25 \%$, which constitute a serious loss in pulse energy. Saturable absorbers can remove ASE in femtosecond dye amplifiers, but their use in CPA systems could induce spectral shift and narrowing of the highly chirped pulses. Also, the ASE overlapped on the stretched pulse would not be removed, leaving a pedestal with a duration of the stretched pulse. Removing the prepulses and the ASE with saturable absorbers after compression would involve considerable energy losses even in the main pulse and degrade the quality of the pulse through nonlinear propagation in the absorber. From the standpoint of solid-target interactions, brute-force methods to clean the pulse include frequency doubling the recompressed pulse, using saturable absorbers and adding extra Pockels' cells in the amplification chain [55]. Another way of diminishing the ASE and prepulse problems is to replace the regenerative amplifier by one or several multipass amplifiers [56].

Using a combination of the methods above, it has been reported that one can improve the ICR to values of $10^{8}-10^{9}$ [55], [56]. We present here two complementary ways to 


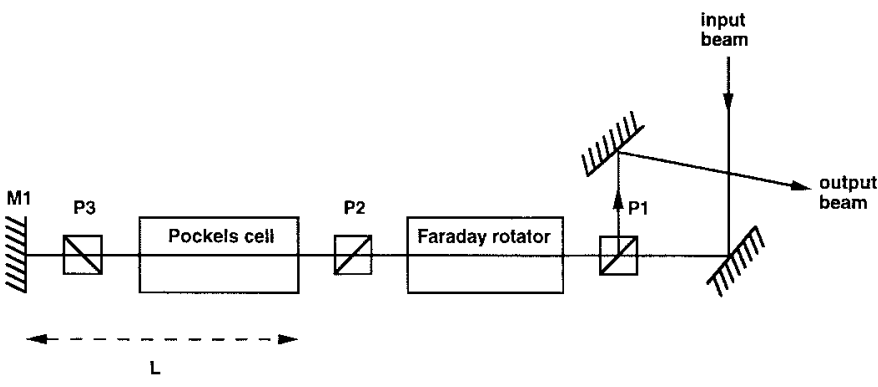

Fig. 6. Schematic representation of a double-pass PC. The input and output beams are collinear going through the PC to ensure a good extinction ratio, and are separated by the Faraday rotator.

improve the ICR of a laser, without major changes to the original system.

\section{A. Double-Pass, High-Contrast Pockels Cell}

The third "brute-force" method listed above to improve the ICR involves PC's. Many CPA laser systems include PC's to bring down the repetition rate from the oscillator for the amplification stages or to remove unwanted prepulses generated in regenerative amplifiers. The use of a single PC will typically produce contrasts of at best $5 \times 10^{3}$ between the switched pulse and the leakage of pulses through the device. In order to produce better contrasts $\left(\approx 10^{7}\right)$, it is common to place two PC's in line [55], but this solution can be rather costly. A less expensive alternative to this approach is to use a single PC twice by reflecting the beam back through the cell. Usually, this would require some misalignement of the $\mathrm{PC}$ to allow for the separation of the incoming and outgoing beams. We show here how we can separate these beams using a Faraday rotator, thus eliminating the extinction ratio degradation which would result from misalignement of the cell.

The double-pass PC system is shown schematically in Fig. 6. The Faraday rotator and polarizers $\mathrm{P} 1$ and $\mathrm{P} 2$ (shared with the PC) define the input polarization for the PC. After an initial pass through the PC, the laser beam is reflected straight back by mirror M1 for the second pass, after which it gets separated from the input beam by the Faraday rotator. The only constraint other than the careful alignment of the direction and polarization of the input and output beams is the distance $L$ of the mirror M1 from the PC. A typical PC temporal window is $T=5-10 \mathrm{~ns}$. As long as the total travel time of the pulse inside the system $(2 L / c$, where $c$ is the speed of light) is shorter than $T$, the PC is active for both passes.

This system has not yet been implemented into a CPA laser chain, but initial tests on this configuration using a continuouswave (CW) YAG laser at $1 \mu \mathrm{m}$ have demonstrated the viability of the technique. A PC with a $\mathrm{LiNbO}_{3}$ crystal was used, with high-quality Glan Laser polarizers. A picowattmeter detected the intensity of the $\mathrm{CW}$ beam. We measured an extinction ratio of $1.6 \times 10^{5}$ for the crossed polarizer without PC. When the PC was added, the extinction ratio decreased to $4 \times 10^{3}$ for a single pass. After double-passing the PC, the extinction ratio was measured to be $2 \times 10^{5}, 3 \mathrm{~m}$ away from the system's output, which constitutes an improvement of 50 over the single-pass configuration. However, we found that the detected

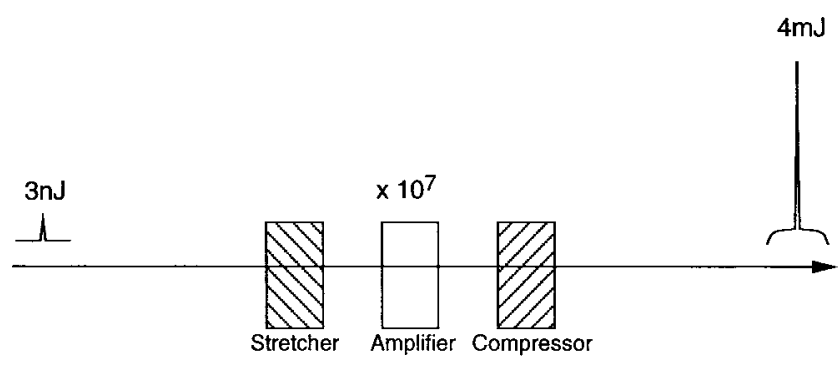

(a)

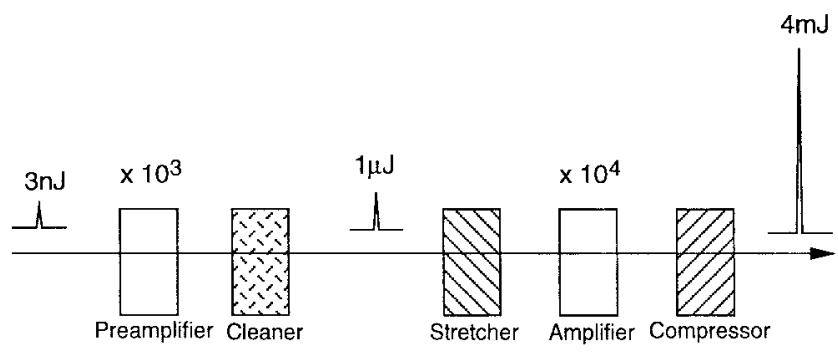

(b)

Fig. 7. Schematics of (a) a typical CPA laser and (b) a modified CPA laser concept with injection of clean microjoule pulses for the suppression of the ASE. The amplification ratios and energies quoted here are those from the CUOS $10-\mathrm{Hz} 100-\mathrm{fs}$ Ti:sapphire laser.

residual light signal was not the system's leakage but in fact due to light back-scattered from the front side of the PC crystal. Indeed, the intensity of this scattered light varied as $1 / d^{2}$, where $d$ is the distance between the front side of the PC and the detector. In a high power laser system, most this scattered light will be filtered away by the amplifiers. This should further improve the extinction ratio to a value between the measured $2 \times 10^{5}$ and the theoretical value of $\left(4 \times 10^{3}\right)^{2}=$ $1.6 \times 10^{7}$ corresponding to a true double-pass into the PC system. The transmission through the system was $20 \%$, but with better mirrors and all surfaces coated for antireflection, a transmission upwards of $50 \%$ can be expected.

\section{B. Clean High-Energy Regenerative Amplifier Seed-Pulse Injection}

While a double-pass PC may improve the prepulse situation in CPA lasers, it does little to increase the contrast in the temporal window of the PC or on the timescale of the stretched pulse (typically $0.2-1.0 \mathrm{~ns}$ ). It is therefore highly desirable to find ways to improve the ASE contrast for short pulses. Techniques that have been devised to clean the pulses include temporal gating [62] and nonlinear discrimination [63], [51], but none of these eliminate the ASE at its source, during the amplification. The method we chose to reduce the ASE in our 10-Hz 100-fs Ti:sapphire laser chain is that of high-energy seed-pulse injection into the amplifier chain [22].

Fig. 7(a) shows a schematic of the typical CPA system. All the amplification-a factor of up to $10^{10}$ or more in the bigger chains - takes place essentially in one step, taking nanojoule pulses from the oscillator to multijoule energies. Along the way, the ASE present in the chain sees as much gain as the main pulse, or more if the laser is run into saturation. Therefore, considerable ASE background remain after compression 
for a low ICR. To reduce the gain seen by the ASE, we make use of a common practice of electronics: preamplification and filtering, as illustrated in Fig. 7(b). The low intensity ultrafast pulses are preamplified-without stretching-to the microjoule level. These pulses have an energy low enough to avoid damaging the preamplifier crystal but high enough to be cleaned when focused into a solid-state saturable absorber ["cleaner" in Fig. 7(b)]. The resulting high-contrast microjoule pulses are then sent into the usual CPA laser chain for the rest of the amplification. This method improves the contrast in two ways: 1) the pulses sent to the CPA chain are cleaner than those coming straight from the oscillator and 2) they need not go through as much amplification in the CPA system for a lower ASE buildup. High-energy seed-pulse injection has been used in the past, but only as applied to Nd:glass-based picosecond systems with nonstationary high-energy oscillators [64], [65]. Our technique is easily applicable to existing subpicosecond CPA laser systems with more conventional oscillators.

The original $10-\mathrm{Hz}$ laser system with which we conducted the research discussed in Sections II and III is composed of: 1) a Clark-MXR self-mode-locked Ti:sapphire oscillator producing 3-nJ 50-fs pulses at $100 \mathrm{MHz}$ with a spectral width of $20 \mathrm{~nm}$ centered at $780 \mathrm{~nm}$; 2) an all reflective sphericaloptics stretcher taking the pulses to 220-ps duration; 3) a saturated regenerative amplifier bringing the pulse energy up to $1 \mathrm{~mJ}$; 4) two multipass amplifiers (2-pass and 4-pass) to further increase the energy to $250 \mathrm{~mJ}$ before compression; and 5 ) a grating compressor to bring the pulse duration back below 100 fs. Two Medox PC's are used as single-pulse-selectors, one right after the oscillator to bring the repetition rate from $100 \mathrm{MHz}$ to $10 \mathrm{~Hz}$, and one after the regenerative amplifier to clean up the prepulses generated in the amplifier [66]. The preamplifier was placed between the first PC and the stretcher. It is a six-pass confocal Ti:sapphire amplifying stage with two concave mirrors and a flat mirror [67]. The crystal $(0.15 \%$ doping, FOM 300) is pumped from both sides with a total of $10 \mathrm{~mJ}$ of green light to achieve a high gain without damage. The preamplified pulses have an energy of typically $3 \mu \mathrm{J}$, and they are focused onto a solid-state saturable absorber (HOYA IR85) to remove the ASE present at this point in the chain. The clean microjoule pulses are then sent to the rest of the original $10-\mathrm{Hz}$ chain.

The ICR of the ultrashort pulses was monitored at different stages of the amplification, for both the "original" and "improved" system, with a high-dynamic-range third-order autocorrelator [68], [22] with up to 1-ns range before and after the main pulse. Autocorrelations with $\approx 10^{8}$ dynamic range were taken of the oscillator, of the preamplified pulse before and after the saturable absorber, and of the compressed pulse after the two-pass amplifier $(10 \mathrm{~mJ}$ before compression, $4 \mathrm{~mJ}$ after). We first show autocorrelations of the oscillator (circles) and of the "original" amplified and compressed pulses (crosses) in Fig. 8. The sharp secondary peaks at 3 and $12 \mathrm{ps}$ are artefacts due to surface reflections in the autocorrelator; negative delay times on the horizontal axis correspond to the front of the pulse. The temporal scale in Fig. 8(b) is logarithmic and concentrates on the front part of the pulse. The oscillator ICR is better than $10^{7}$ on a temporal scale

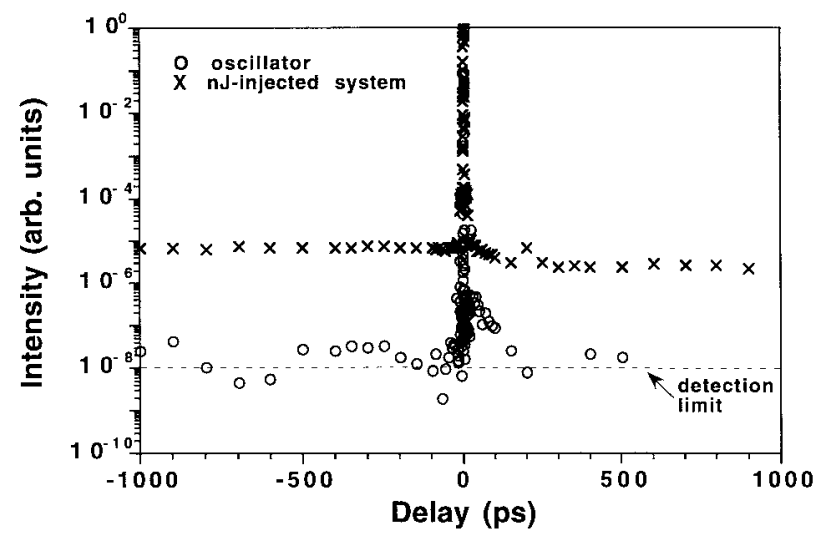

(a)

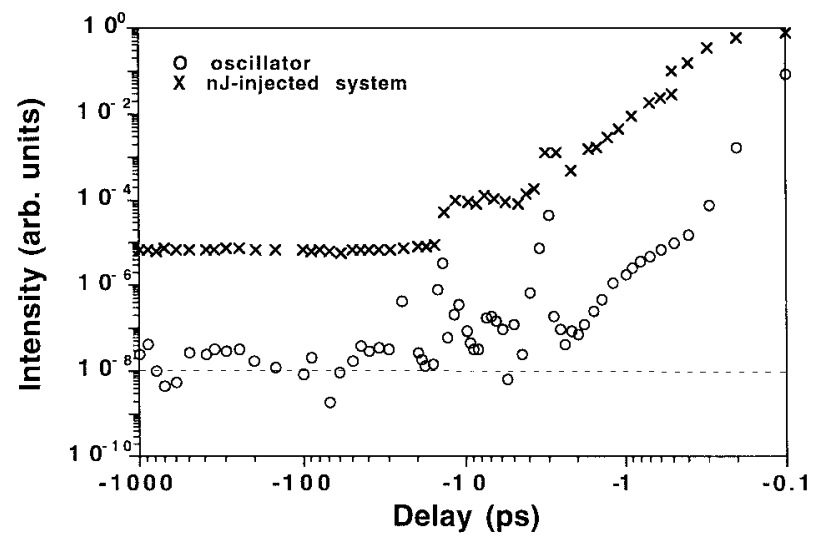

(b)

Fig. 8. High-dynamic-range third-order autocorrelation traces of the pulses from the oscillator (open circles) and the original $10-\mathrm{Hz}$ laser (crosses); negative direction of the delay corresponds to the leading edge of the pulse. (a) In a linear temporal scale and (b) in a logarithmic temporal scale showing the front part of the pulse.

longer than $10 \mathrm{ps}$, and this is what the system starts with. After amplification in the "original" system, the contrast is now about $10^{5}$ in the nanosecond timescale, in agreement with the plasma-shuttered streak camera measurement shown in Section III-A. The trace of the amplified pulse features shoulders in the first $10 \mathrm{ps}$; these are probably due to the uncompensated residual phase. Considering that the pulses are stretched to $220 \mathrm{ps}$ during amplification, the contrast of $10^{5}$ that extends to at least one nanosecond is not due to phase distortion but to the ASE. The third-order autocorrelations of the amplified pulse shows a FWHM duration of $300 \mathrm{fs}$, due to the material dispersion and the group velocity mismatch in the nonlinear crystals; single-shot autocorrelations of the pulses indicate an actual duration of $\approx 70$ ps. The real ICR is, therefore, higher than that measured by the autocorrelation trace by a factor of about 5 .

Fig. 9 shows the ICR measurement for the compressed pulses from the preamplifier without the saturable absorber (circles) and from the "improved" system, including cleaning and amplification up to $4 \mathrm{~mJ}$ (crosses). The preamplified pulse without cleaning has an ICR of $\approx 5 \times 10^{7}$ on the nanosecond scale, while the "improved" system has an ICR of $10^{7}$. The microjoule pulses actually have a better contrast than shown in Fig. 9 upon injection through the system due to the 


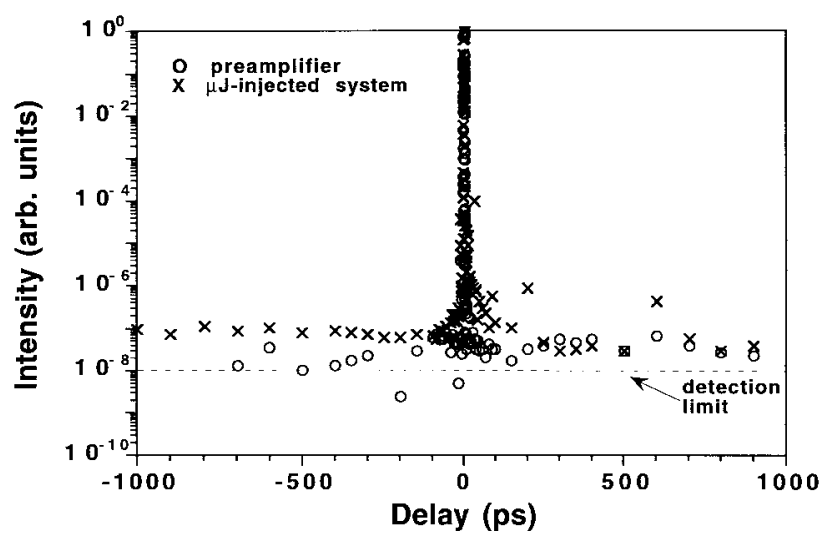

(a)

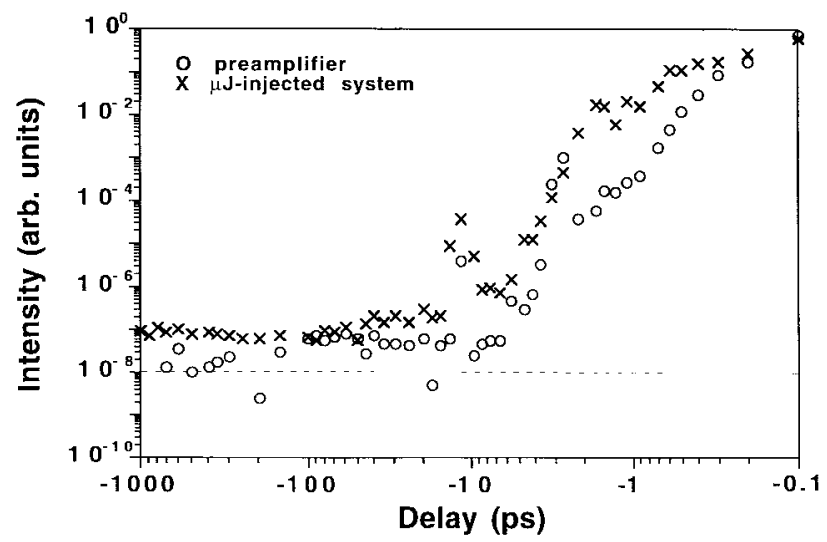

(b)

Fig. 9. High-dynamic-range third-order autocorrelation traces of the pulses from the preamplifier (open circles) and the improved $10-\mathrm{Hz}$ laser injected with clean $\mu \mathrm{J}$ pulses (crosses); negative direction of the delay correspond to the leading edge of the pulse. (a) In a linear temporal scale and (b) in a logarithmic temporal scale showing the front part of the pulse.

cleaning, which improved the ICR of the preamplified pulse. Because the ASE background is significantly suppressed by the saturable absorber, it was not measurable with the thirdorder autocorrelator. We can nevertheless estimate the ICR of the clean microjoule pulse from the transmission of the main pulse and the ASE measured with a fast photodiode: 0.5 and $4 \times 10^{-3}$, respectively. The contrast of the pulse right after the saturable absorber is then $5, \times 10^{7} /\left[\left(4 \times 10^{-3}\right) / 0.5\right] \approx$ $6 \times 10^{9}$. Because these clean pulses were at the microjoule level, we were able to reduced the number of passes in the regenerative amplifier and limit the growth of the ASE background in the chain. This method could give even higher contrasts if the unstretched pulse could be amplified to higher energies $(>10 \mu \mathrm{J})$ to take better advantage of the saturable absorber, but care must be taken to remain below the damage threshold of the preamplifier optics. With the present setup, injecting clean high-contrast microjoule pulses into the $10-\mathrm{Hz}$ CPA laser chain has improved its ICR by at least a factor 100 , from $10^{5}$ to $10^{7}$. This improvement came relatively cheaply (one low-energy multipass amplifier), with no loss in the final pulse energy and with minimal changes to the original laser system (a readjustment of the regenerative amplifier timing). Laser-matter interaction experiments conducted using this new and improved $10-\mathrm{Hz}$ laser show very high plasma densities, and have allowed to study the atomic physics of strongly coupled plasmas in regimes that could not have been reached with the original low-contrast system [46], [47].

\section{CONCLUSION}

We have discussed and emphasized the importance of the laser ICR for ultrafast laser-solid interactions. We have shown the damaging effect that a poor contrast can have on three different basic and technological research projects using 100-fs laser pulses: the generation and probing of shocked solid matter, the acceleration of ions from solid targets and the development of jitter-free ultrafast detectors. We then introduced a novel pulse-contrast measurement technique, the high-dynamic-range plasma-shuttered streak camera, which extends the dynamic range of a picosecond visible streak camera from the usual 100 to a demonstrated $10^{7}$, with possible improvement to $10^{9}$. We measured the ICR of a 10 $\mathrm{Hz}$ 100-fs Ti:sapphire laser over the nanosecond range with this new diagnostics, and the obtained value of $10^{5}$ agrees with a third-order autocorrelation of the same system.

To improve this contrast for better solid-target interactions, we proposed and tested two new methods: the double-pass, high-contrast PC, and high-energy clean seed-pulse injection of the $10-\mathrm{Hz}$ laser system. Initial demonstration experiments on the double-pass PC showed an improvement factor of 50 over the single-pass configuration, with an obvious possible extension up to a total double-pass extinction ratio of $1.6 \times 10^{7}$. On the $10-\mathrm{Hz}$ laser, we have suppressed the ASE level by more than a factor of 100 by injecting high-energy seed-pulses into the existing system. The clean microjoule pulses were obtained with a simple six-pass preamplifier and a solid-state saturable absorber. Both techniques are relatively low-cost methods of improving the ICR of existing Ti:sapphire laser systems.

\section{ACKNOWLEDGMENT}

The authors would like to thank J.B. Workman and A. Maksimchuk for fruitful discussions, as well as K. Read for sample preparation and Kaiser Optical System Inc. for providing the photopolymer sample for the plasma-shuttered streak camera measurements. Finally, they would like to acknowledge Spectrogon Inc. for supplying the compressor gratings for the $10-\mathrm{Hz} 100-$ fs laser system.

\section{REFERENCES}

[1] D. Strickland and G. Mourou, "Compression of amplified chirped optical pulses," Opt. Commun., vol. 56, pp. 219-221, 1985.

[2] P. Maine, D. Strickland, P. Bado, M. Pessot, and G. Mourou, "Generation of ultrahigh peak power pulses by chirped pulse amplification," IEEE J. Ouantum Electron., vol. 24, pp. 398-403, 1988.

[3] C. Rouyer, E. Mazataud, I. Allais, A. Pierre, S. Seznec, C. Sauteret, G. Mourou, and A. Migus, "Generation of 50-TW femtosecond pulses in a Ti:sapphire/Nd:glass chain,” Opt. Lett., vol. 18, pp. 214-216, 1993.

[4] M. D. Perry and G. Mourou, "Terawatt to petawatt subpicosecond lasers," Science, vol. 264, pp. 917-924, 1994.

[5] C. P. J. Barty, C. L. Gordon, III, and B. E. Lemoff, "Multiterawatt 30-fs Ti:sapphire laser system," Opt. Lett., vol. 19, p. 1442-1444, 1994.

[6] J. J. Macklin, J. D. Kmetec, and C. L. Gordon III, "High-order harmonic generation using intense femtosecond pulses," Phys. Rev. Lett., vol. 70, pp. 766-769, 1993. 
[7] A. L'Huillier, T. Auguste, P. Balcou, B. Carre, P. Monot, P. Salières, C. Altucci, M. B. Gaarde, J. Larsson, E. Mevel, T. Starczewski, S. Svanberg, C.-G. Whalstrom, R. Zerne, K. S. Budil, T. Ditmire, and M D. Perry, "High-order harmonics: A coherent source in the XUV range," J. Nonlin. Opt. Phys. Mater., vol. 4, pp. 647-665, 1995.

[8] Z. Chang, A. Rundquist, H. Wang, H. C. Kapteyn, and M. M. Murnane, "Generation of coherent soft X-rays at $2.7 \mathrm{~nm}$ using high harmonics," Phys. Rev. Lett., vol. 79, pp. 2967-2970, 1997.

[9] C. A. Coverdale, C. B. Darrow, C. D. Decker, W. B. Mori, K.-C. Tzeng, K. A. Marsh, C. E. Clayton, and C. Joshi, "Propagation of intense subpicosecond laser pulses through underdense plasmas," Phys. Rev. Lett., vol. 74, pp. 4659-4662, 1995.

[10] K. Nakajima, D. Fisher, T. Kawakubo, H. Nakanishi, A. Ogata, Y. Kato, Y. Kitagawa, R. Kodama, K. Mima, H. Shiraga, K. Suzuki, K. Yamakawa, T. Zhang, Y. Sakawa, T. Shoji, N. Yugami, M. Downer, and T. Tajima, "Observation of ultrahigh gradient electron acceleration by a self-modulated intense short laser pulse," Phys. Rev. Lett., vol. 74 pp. 4428-4431, 1995.

[11] A. Modena, Z. Najmudin, A. E. Dangor, C. E. Clayton, K. A. Marsh, C. Joshi, V. Malka, C. B. Darrow, C. Danson, D. Neely, and F. N. Walsh, "Electron acceleration from the breaking of relativistic plasma waves," Nature, vol. 377, pp. 606-608, 1995.

[12] D. Umstadter, S.-Y. Chen, A. Maksimchuk, G. Mourou, and R. Wagner, "Nonlinear optics in relativistic plasmas and laser wake field acceleration of electrons," Science, vol. 273, pp. 472-475, 1996.

[13] R. Wagner, S.-Y. Chen, A. Maksimchuk, and D. Umstadter, "Electron acceleration by a laser wakefield in a relativistically self-guided channel," Phys. Rev. Lett., vol. 78, pp. 3125-3128, 1997.

[14] T. Ditmire, J. W. G. Tisch, E. Springate, M. B. Mason, N. Hay, R. A. Smith, J. Marangos, and M. H. R. Hutchinson, "High-energy ions produced in explosions of superheated atomic clusters," Nature, vol. 386, pp. 54-56, 1997.

[15] M. Murnane, H. C. Kapteyn, and R. W. Falcone, "High-density plasmas produced by ultrafast laser pulses," Phys. Rev. Lett., vol. 62, pp. 155-158, 1989.

[16] A. Rousse, P. Audebert, J. P. Geindre, F. Falliès, J. C. Gauthier, A. Mysyrowicz, G. Grillon, and A. Antonetti, "Efficient $K$ alpha X-ray source from femtosecond laser-produced," Phys. Rev. E, vol. 50, pp. 2200-2207, 1994.

[17] Z. Jiang, J. C. Kieffer, J. P. Matte, M. Chaker, O. Peyrusse, D. Gilles, G. Korn, A. Maksimchuk, S. Coe, and G. Mourou, "X-ray spectroscopy of hot solid density plasmas produced by subpicosecond high contrast laser pulses at $10^{18}-10^{19} \mathrm{~W} / \mathrm{cm}^{2}$," Phys. Plasma, vol. 2, pp. 1702-1711, 1995

[18] J. Workman, A. Maksimchuk, X. Liu, U. Ellenberger, J. S. Coe, C. Y. Chien, and D. Umstadter, "Control of bright picosecond X-ray emission from intense subpicosecond laser-plasma interactions," Phys. Rev. Lett., vol. 75, pp. 2324-2327, 1995.

[19] P. A. Norreys, M. Zepf, S. Moustaizis, A. P. Fews, J. Zhang, P. Lee, M. Bakarezos, C. N. Danson, A. Dyson, P. Gibbon, P. Loukakos, D. Neely, F. N. Walsh, J. S. Wark, and A. E. Dangor, "Efficient extreme UV harmonics generated from picosecond laser pulse interactions with solid targets," Phys. Rev. Lett., vol. 76, pp. 1832-1835, 1996.

[20] P. A. VanRompay, M. Nantel, and P. P. Pronko, "Pulse-contrast effects on energy distributions of $\mathrm{C}^{1+}-\mathrm{C}^{4+}$ ions for high-intensity $100-\mathrm{fs}$ laser-ablation plasmas," in press, Appl. Surf. Sci., 1998.

[21] A.-C. Tien, M. Nantel, G. Mourou, D. Kaplan, and M. Bouvier, "High-dynamic-range laser-pulse-contrast measurement with a plasmashuttered streak camera," Opt. Lett., vol. 22, pp. 1559-1561, 1997.

[22] J. Itatani, J. Faure, M. Nantel, G. Mourou, and S. Watanabe, "Suppression of the amplified spontaneous emission in chirped-pulseamplification lasers by clean high-energy seed-pulse injection," Opt. Commun., to be published.

[23] Ph. Zeitoun, A. Carillon, A. Demir, E. Fill, M. Holden, P. Jaeglé, G. Jamelot, A. Klisnick, J. Krisnan, C. Lewis, M. Nantel, A. McPhee, B. Rus, D. Schlögl, and G. Tallents, "Reduction of X-ray laser driver energy," J. Electron Spectrosc. Relat. Phenom., vol. 80, pp. 255-258, 1996.

[24] D. Du, X. Liu, G. Korn, J. Squier, and G. Mourou, "Laser-induced breakdown by impact ionization in $\mathrm{SiO}_{2}$ with pulse widths from $7 \mathrm{~ns}$ to $150 \mathrm{fs,"} \mathrm{Appl.} \mathrm{Phys.} \mathrm{Lett.,} \mathrm{vol.} \mathrm{64,} \mathrm{pp.} \mathrm{3071-3073,} 1994$.

[25] S. Ichimaru, "Strongly-coupled plasmas: high-density classical plasmas and degenerate electron liquids," Rev. Mod. Phys., vol. 54, pp $1017-1059,1982$

[26] C. M. Lee and A. Hauer, "Measurements of compressed core density of laser-imploded targets by X-ray continuum-edge shift," Appl. Phys. Lett., vol. 33, pp. 692-694, 1978.
[27] B. A. Hammel, C. J. Keane, M. D. Cauble, D. R. Kania, J. D. Kilkenny, R. W. Lee, and R. Pasha, "X-ray spectroscopic measurements of high densities and temperatures from indirectly driven inertial confinement fusion capsules," Phys. Rev. Lett., vol. 70, pp. 126312-66, 1993.

[28] L. DaSilva, A. Ng, B. K. Godwal, G. Chiu, and F. Cottet, "Shockinduced shifts in the aluminum K photoabsorption edge," Phys. Rev. Lett., vol. 62, pp. 1623-1626, 1989.

[29] A. Djaoui, T. A. Hall, R. Eason, C. Jackson, and S. J. Rose, "Interpretation of EXAFS data from laser shock compressed plasmas," Plasma Phys. Controlled Fus., vol. 31, pp. 111-122, 1989.

[30] J. Workman, M. Nantel, A. Maksimchuk, and D. Umstadter, "Application of a picosecond soft X-ray source to time-resolved plasma dynamics," Appl. Phys. Lett. vol. 70, pp. 312-314, 1997.

[31] R. Evans, A. D. Badger, F. Falliès, M. Mahdieh, T. A. Hall, P. Audebert, J.-P. Geindre, J.-C. Gauthier, A. Mysyrowicz, G. Grillon, and A. Antonetti, "Time- and space-resolved optical probing of femtosecondlaser-driven shock waves in aluminum," Phys. Rev. Lett., vol. 77, pp. 3359-3362, 1996.

[32] V. E. Gusev, "Estimate of parameters for shock waves excited by femtosecond laser pulses," Phys. Vibr., vol. 57, pp. 1-10, 1993.

[33] A. Ng, A. Forsman, and P. Celliers, "Heat front propagation in femtosecond-laser-heated solids," Phys. Rev. E, vol. 51, pp. R5208-R5211, 1995.

[34] J. Workman, A. Maksimchuk, X. Liu, U. Ellenberger, J. S. Coe, C.-Y. Chien, and D. Umstadter, "Picosecond soft-X-ray source from subpicosecond laser-produced plasmas," J. Opt. Soc. Amer. B, vol. 13, pp. $125-131,1996$

[35] A. J. Peurrung, J. P. Cowin, G. Teeter, S. E. Barlow, and T. M. Orlando, "Space-charge-induced acceleration of ions emitted by laser-irradiated surfaces," J. Appl. Phys., vol. 78, pp. 481-488, 1995.

[36] S. C. Wilks, "Simulations of ultraintense laser-plasma interactions," Phys. Fluids B, vol. 5, pp. 2603-2608, 1993.

[37] E. G. Gamaly, "The ponderomotive force in an overdense, absorbing, dispersive plasma caused by an intense laser-matter interaction," Phys. Fluids B, vol. 5, pp. 3765-3769, 1993.

[38] S. V. Bulanov and N. M. Naumova, "Interaction of an ultrashort, relativistically strong laser pulse with an overdense plasma," Phys. Plasma, vol. 1, pp. 745-757, 1994

[39] L. Laska, J. Krasa, K. Masek, M. Pfeifer, P. Trenda, B. Kralikova, J. Skala, K. Rohlena, E. Wyrona, J. Farny, P. Parys, J. Wolowski, W. Mroz, A. Shumshurov, B. Sharkov, J. Collier, K. Langbein, and H. Haseroth, "Multiply charged ion generation from NIR and visible laser-produced plasma," Rev. Sci. Instrum., vol. 67, pp. 950-952, 1996.

[40] F. Qian, R. K. Singh, S. Dutta, P. P. Pronko, and W. H. Weber, "Femtosecond laser deposition of diamond-like carbon films," Proc. Symp. Mater. Res. Soc., 1996, vol. 397, pp. 297-302.

[41] G. Guethlein, J. Bonlie, D. Price, R. Shepherd, B. Young, and R. Stewart, "Charge and mass resolved time of flight observations of 140 fs laser produced ions," Rev. Sci. Instrum., vol. 66, pp. 333-335, 1995.

[42] P. A. VanRompay, M. Nantel, and P. P. Pronko, "Carbon ion charge states and energy distributions for DLC ablation plumes from 100-fs 780-nm laser pulses," in press, Surf. Coat. Technol., 1998.

[43] W. Knox and G. Mourou, "A simple jitter-free picosecond streak camera," Opt. Commun., vol. 37, pp. 203-206, 1981.

[44] A. Maksimchuk, M. Kim, J. Workman, G. Korn, J. Squire, D. Du, D. Umstadter, G. Mourou, and M. Bouvier, "Signal averaging X-ray streak camera with picosecond jitter," Rev. Sci. Instrum., vol. 67, pp. 697-699, 1996.

[45] C. Y. Côté, J. C. Kieffer, P. Gallant, J. C. Rebuffie, C. Goulmy, A Maksimchuk, G. Mourou, D. Kaplan, and M. Bouvier, in SPIE Proc., vol. 2869 , p. $956,1997$.

[46] M. Nantel, G. Ma, S. Gu, C. Y. Côté, J. Itatani, T. Buma, A. Maksimchuk, and D. Umstadter, "Experimental study of continuum lowering," SPIE Proc., vol. 3157, p. 93, 1997.

[47] M. Nantel, G. Ma, S. Gu, C. Y. Côté, J. Itatani, and D. Umstadter, "Pressure ionization and microfields in strongly-coupled Coulomb systems," Phys. Rev. Lett., May 1998

[48] A. Braun, J. V. Rudd, H. Cheng, G. Mourou, D. Kopf, I. D. Jung, K. J. Weingarten, and U. Keller, "Characterization of short-pulse oscillators by means of a high-dynamic-range autocorrelation measurement," Opt. Lett., vol. 20, pp. 1889-1891, 1995.

[49] P. F. Curley, G. Darpentigny, G. Cheriaux, J. P. Chamberet, and A. Antonetti, "High dynamic range autocorrelation studies of a femtosecond Ti:sapphire oscillator and its relevance to the optimization of chirped pulse amplification systems," Opt. Commun., vol. 120, pp. 71-77, 1995.

[50] B. C. Stuart, M. D. Feit, A. M. Rubenchik, B. W. Shore, and M. D. Perry, "Laser-induced damage in dielectrics with nanosecond to subpicosecond pulses," Phys. Rev. Lett., vol. 74, pp. 2248-2251, 1995. 
[51] H. C. Kapteyn, M. M. Murnane, A. Szoke, and R. W. Falcone, "Prepulse energy suppression for high-energy ultrashort pulses using self- induced plasma shuttering," Opt. Lett. vol. 16, pp. 490-492, 1991.

[52] A. Zigler, "Plasma production from ultraviolet-transmitting targets using subpicosecond ultraviolet radiation," Opt. Lett., vol. 16, pp. 1261-1263, 1991.

[53] S. Backus, H. C. Kapteyn, M. M. Murnane, D. M. Gold, H. Nathel, and W. White, "Prepulse suppression for high-energy ultrashort pulses using self-induced plasma shuttering from a fluid target," Opt. Lett., vol. 18, pp. 134-136, 1993.

[54] D. M. Gold, "Direct measurement of prepulse suppression by use of a plasma shutter," Opt. Lett., vol. 19, pp. 2006-2008, 1994.

[55] D. F. Price, R. M. More, R. S. Walling, G. Guethlein, R. L. Shepherd, R. E. Stewart, and W. E. White, "Absorption of ultrafast laser pulses by solid targets heated rapidly to temperatures $1-1000 \mathrm{eV}$," Phys. Rev. Lett., vol. 75, pp. 252-55, 1995.

[56] A. Antonetti, F. Blasco, J. P. Chambéret, G. Chériaux, G. Darpentigny, C. Le Blanc, P. Rousseau, S. Ranc, and F. Salin, "A laser system producing $5 \times 10^{19} \mathrm{~W} / \mathrm{cm}^{2}$ at $10 \mathrm{~Hz}, "$ Appl. Phys. B, vol. 65 , pp. 197-204, 1997.

[57] B. E. Lemoff and C. P. J. Barty, "Quintic-phase-limited, spatially uniform expansion and recompression of ultrashort optical pulses," Opt. Lett., vol. 18, pp. 1651-1653, 1993.

[58] W. E. White, F. G. Patterson, R. L. Combs, D. F. Price, and R. L. Shepherd, "Compensation of higher-order frequency-dependent phase terms in chirped-pulse amplification systems," Opt. Lett., vol. 18, pp. $1343-1345,1993$

[59] S. Backus, C. G. Durfee, III, G. Mourou, H. C. Kapteyn, and M. M. Murnane, "0.2-TW laser system at $1 \mathrm{kHz}$," Opt. Lett., vol. 22, pp. $1256-1258,1997$.

[60] S. Backus, C. G. Durfee, III, H. C. Kapteyn, and M. M. Murnane, Rev. Sci. Intrum., to be published.

[61] C. Y. Chien, Ph.D. dissertation, Univ. of Michigan, unpublished, 1993.

[62] K. Yamakawa, H. Shiraga, Y. Kato, and C. P. J. Barty, "Prepulse-free 30-TW, 1-ps Nd:glass laser," Opt. Lett., vol. 16, pp. 1593-1595, 1991.

[63] J.-L. Tapié and G. Mourou, "Shaping of clean, femtosecond pulses at 1.053 microns for chirped-pulse amplifications," Opt. Lett., vol. 17, pp. 136-138, 1992.

[64] R. S. Marjoribanks, F. W. Budnik, L. Zhao, G. Kulcsár, M. Stanier, and J. Mihaychuk, "High-contrast terawatt chirped-pulse-amplification laser that uses a 1-ps Nd:glass oscillator," Opt. Lett., vol. 18, pp. 361-363, 1993.

[65] F. Billhardt, M. Kalashnikov, P. V. Nickles, and I. Will, "A highcontrast ps-terawatt Nd:glass laser system with fiberless chirped pulse amplification," Opt. Commun., vol. 98, pp. 99-104, 1993.

[66] D. Du, J. Squier, S. Kane, G. Korn, G. Mourou, C. Bogush, and C. Cotton, "Terawatt Ti:sapphire laser with a spherical reflective-optic pulse expander," Opt. Lett., vol. 20, pp. 2114-2116, 1995.

[67] S. Backus, J. Peatross, C. P. Huang, M. M. Murnane, and H. C. Kapteyn, "Ti:sapphire amplifier producing millijoule-level, 21-fs pulses at $1 \mathrm{kHz}$," Opt. Lett., vol. 20, pp. 2000-2002, 1995.

[68] G. Albrecht, A. Antonetti, and G. Mourou, "Temporal shape analysis of Nd:YAG active passive mode-locked pulses," Opt. Commun., vol. 40, pp. 59-62, 1981.

Marc Nantel, for a biography, see this issue, p. 384.

Jiro Itatani, photograph and biography not available at the time of publication.

An-Chun Tien received the B.S. and M.S. degrees in physics from National Taiwan University, Taiwan, R.O.C., in 1991 and 1989, respectively, and is currently working toward the Ph.D. degree at the Center for Ultrafast Optical Science (CUOS), University of Michigan at Ann Arbor.

$\mathrm{He}$ is a Graduate Student Research Assistant at CUOS. He is currently investigating the mechanism for laser-induced damage in wide-gap materials.
Jérôme Faure, for a biography, see this issue, p. 383

Daniel Kaplan is a specialist in the physics of semiconductors, has made a 25year career in industrial research, involving work at IBM T.J. Watson Research Center, Thomson CSF Research Laboratory and the Compagnie Généerale de Radiologie. A frequent scientific visitor to the University of Michigan, he has been involved in several experiments concerning photoconductivity induced by picosecond lasers. As a former president of the French Physical Society he has been a strong advocate of enhancing ties between Academia and Industry and is now operating a small company, Alliage, to put his ideas into practice.

Marcel Bouvier was born in Albertville, France, on May 2, 1946. He received the Maitrise EEA (Electronique, Electrotechnique, Automatisation) degree from the University of Grenoble, Grenoble, France.

From 1984 to 1988, he was a Research Engineer at the University of Rochester. From 1988 to 1990, he was a Research Engineer at the University of Michigan at Ann Arbor. In 1985, he co-founded Medox Electro-Optics Inc., Ann Arbor, MI, and has been acting as its President since 1990.

Takashi Buma received the B.S.E. degree in electrical engineering from Princeton University, Princeton, NJ, in 1995 , and is currently working toward the Ph.D. degree in applied physics at the University of Michigan at Ann Arbor.

His research interests are ultrasound imaging and optical methods for generating and detecting ultrasound.

Paul Van Rompay, photograph and biography not available at the time of publication.

John Nees, for a biography, see this issue, p. 383.

Peter P. Pronko is a Research Scientist and the Associate Director for Industry Liaison at the Center for Ultrafast Optical Science at the University of Michigan. His professional activities involve the use of ultrafast lasers in basic and applied materials research and the investigation of their various applications to industrial interests.

Donald Umstadter, photograph and biography not available at the time of publication.

Gérard A. Mourou (M'89-SM'97-F'98), for a biography, see this issue, p. 384 . 